\title{
Molecular Dynamics Simulation Studies of Viscosity and Diffusion of $n$-Alkane Oligomers at High Temperatures
}

\author{
Song Hi Lee \\ Department of Chemistry, Kyungsung University, Busan 608-736, Korea. *E-mail: shlee@ks.ac.kr \\ Received July 28, 2011, Accepted August 31, 2011
}

\begin{abstract}
In this paper we have carried out molecular dynamics simulations (MD) for model systems of liquid $n$-alkane oligomers $\left(\mathrm{C}_{12} \sim \mathrm{C}_{80}\right)$ at high temperatures $(\sim 2300 \mathrm{~K})$ in a canonical ensemble to calculate viscosity $\eta$, selfdiffusion constants $\mathrm{D}$, and monomeric friction constant $\zeta$. We found that the long chains of these $n$-alkanes at high temperatures show an abnormality in density and in monomeric friction constant. The behavior of both activation energies, $E_{\eta}$ and $E_{D}$, and the mass and temperature dependence of $\eta, D$, and $\zeta$ are discussed.
\end{abstract}

Key Words : MD simulation, Diffusion, Viscosity, Friction, $n$-Alkane

\section{Introduction}

A number of studies have been reported on the relationship between the zero shear viscosity $\left(\eta_{0}\right)$ and molecular weight. For linear polymers, $\eta_{0}$ increases linearly with the molecular weight M. ${ }^{1}$ At values of molecular weight greater than a critical molecular weight, $\mathrm{M}_{\mathrm{c}}, \eta_{0}$ becomes proportional to $\mathrm{M}^{3.4}$ proposed in 1951 by Fox and Flory ${ }^{2}$ on the basis of measurements on narrow distribution fractions of polystyrene and polyisobutylene. Self-diffusion constant (D) of polymer melts have also shown a similar trend: $\mathrm{D} \sim \mathrm{M}^{-1}$ for $\mathrm{M}<\mathrm{M}_{\mathrm{c}}$ and $\mathrm{D} \sim \mathrm{M}^{-2}$ for $\mathrm{M}>\mathrm{M}_{\mathrm{c}} . \mathrm{M}_{\mathrm{c}}$ is called the entanglement coupling molecular weight. Below and above $\mathrm{M}_{\mathrm{c}}$, the polymer chain dynamics of unentangled and entangled chains are commonly described by the Rouse model and the reptation model. ${ }^{1,3,4}$

At the molecular weight below the Rouse regime, $\mathrm{D}$ and $\eta$ of $n$-alkanes also show power law behaviors. For example, $\mathrm{D}$ of $n$-alkanes from $n$-octane to polyethylene of the molecular weight of several thousands was reported to follow the relation, $\mathrm{D} \sim \mathrm{M}^{-\alpha}$ in which the exponents $\alpha$ is in the range of 2.72-1.85 depending on temperature..$^{5-7}$ Although the power law dependence has the same functional form as in the Rouse and reptation regimes, the origin is totally different. The molecular weight dependence of $D$ and $\eta$ in the Rouse and reptation regimes is attributed to the topological entanglement effect not to the local segmental friction while the exponent found below the Rouse regime reflects the molecular weight dependence of the local friction rather than the topological effect.

While the Rouse-reptation crossover has received considerable attention in recent years, ${ }^{8,9}$ the $n$-alkane-Rouse crossover has received relatively little attention. The equilibrium (EMD) and non-equilibrium molecular dynamics (NEMD) simulations ${ }^{10-12}$ on the system are useful for the study on the transport properties of liquid $n$-alkanes of moderate molecular sizes $\left(\mathrm{C}_{10}-\mathrm{C}_{40}\right)$, which can shed light on the polymer dynamics in the molecular level.

In this study, we use the Green-Kubo and Einstein formulas for the calculation of the viscosities and diffusion constants of liquid $n-\mathrm{C}_{12} \mathrm{H}_{26}$ to $n-\mathrm{C}_{80} \mathrm{H}_{162}$ using EMD simulation. The main purpose of this study is to characterize the viscosity and self-diffusion dynamics of small $n$-alkanes as a function of chain length $\mathrm{n}$ (or molecular weight $\mathrm{M}$ ) especially at high temperatures.

\section{Molecular Models and MD Simulation Methods}

For liquid $n$-alkanes, we have chosen 7 systems - $n$ dodecane $\left(\mathrm{C}_{12} \mathrm{H}_{26}\right)$, n-eicosane $\left(\mathrm{C}_{20} \mathrm{H}_{42}\right)$, n-octaconsane $\left(\mathrm{C}_{28} \mathrm{H}_{58}\right)$, $n$-hexatriacontane $\left(\mathrm{C}_{36} \mathrm{H}_{74}\right)$, and $n$-tetratetracontane $\left(\mathrm{C}_{44} \mathrm{H}_{90}\right)$, $n$-hexacontane $\left(\mathrm{C}_{60} \mathrm{H}_{122}\right)$, and $n$-octacontane $\left(\mathrm{C}_{80} \mathrm{H}_{162}\right)$. Each simulation was carried out in the $\mathrm{NpT}$ ensemble to determine the volume of each system at given temperatures, and after the equilibrium density and hence the length of cubic simulation box were obtained, new NVT MD simulation was performed for each system to store the configurations of $n$-alkanes for later analyses. The usual periodic boundary condition in the $\mathrm{x}-, \mathrm{y}-$, and $\mathrm{z}$-directions and the minimum image convention for pair potential were applied. Gaussian isokinetics was used to keep the temperature of the system constant. $^{13,14}$

We used a united atom (UA) model for $n$-alkanes, that is, methyl and methylene groups are considered as spherical interaction sites centered at each carbon atom. This model have been used in the previous simulation studies. ${ }^{10-12}$ Here, we briefly describe the salient features of the model. The interaction between the sites on different $n$-alkane molecules and between the sites separated by more than three bonds in the same $n$-alkane molecule is described by a Lennard-Jones (LJ) potential. All the sites in a chain have the same LJ size parameter $\sigma_{\mathrm{i}} \equiv \sigma_{\mathrm{ii}}=3.93 \mathrm{~A}$, the well depth parameters were $\varepsilon_{\mathrm{i}} \equiv \varepsilon_{\mathrm{ii}}=0.94784 \mathrm{~kJ} / \mathrm{mol}$ for interactions between the end sites and $\varepsilon_{\mathrm{i}}=0.39078 \mathrm{~kJ} / \mathrm{mol}$ for interactions between the internal sites. The Lorentz-Berthelot combining rules $\left[\varepsilon_{\mathrm{ij}} \equiv\left(\varepsilon_{\mathrm{i}} \varepsilon_{\mathrm{j}}\right)^{1 / 2}\right.$, $\left.\sigma_{\mathrm{ji}}=\left(\sigma_{\mathrm{i}}+\sigma_{\mathrm{j}}\right) / 2\right]$ were used for interactions between an end site and an internal site. A cut-off distance of $2.5 \sigma_{i}$ was used for all the LJ interactions. 
Initially in NpT MD simulations, the bond-stretching was described by a harmonic potential, with an equilibrium bond distance of $1.54 \mathrm{~A}$ and a force constant of $1882.8 \mathrm{~kJ} / \mathrm{mol} / \mathrm{A}^{2}$. The bond bending interaction was also described by a harmonic potential with an equilibrium angle of $114^{\circ}$ and a force constant of $0.079187 \mathrm{~kJ} / \mathrm{mol} /$ degree. $^{2}$ The torsional interaction was described by the potential developed by Jorgensen et al: $:^{15}$

$$
\mathrm{U}_{\text {torsion }}(\phi)=\mathrm{a}_{0}+\mathrm{a}_{1} \cos \phi+\mathrm{a}_{2} \cos ^{2} \phi+\mathrm{a}_{3} \cos ^{3} \phi,
$$

where $\phi$ is the dihedral angle, and $\mathrm{a}_{0}=8.3973 \mathrm{~kJ} / \mathrm{mol}, \mathrm{a}_{1}=$ $16.7862 \mathrm{~kJ} / \mathrm{mol}, \mathrm{a}_{2}=1.1339 \mathrm{~kJ} / \mathrm{mol}$, and $\mathrm{a}_{3}=-26.3174 \mathrm{~kJ} /$ mol. For the time integration of the equations of motion, we adopted Gear's fifth-order predictor-corrector algorithm ${ }^{16}$ with a time step of 2 femto-second for all the systems. Later in NVT MD simulations, the bond-stretching was switched to a constraint force which keeps intramolecular nearest neighbors at a fixed distance. The advantage for this change is to increase the time step as 5 femto-seconds with the use of RATTLE algorithm ${ }^{17}$ and velocity Verlet algorithm. ${ }^{18}$ After a total of 1,000,000 time steps (5 nano-seconds) for equilibration, the equilibrium properties were then averaged over 5 blocks of 200,000 time steps ( 1 nano-seconds). The configurations of all the molecules for further analyses were stored every 10 time steps ( 0.05 pico second) which is small enough for the tick of any time auto-correlation functions.

Self-diffusion constant is obtained from the Einstein relation of the mean square displacement (MSD): ${ }^{19}$

$$
\mathrm{D}=\frac{1}{6} \lim _{t \rightarrow \infty} \frac{d}{d t}<\left[\mathbf{r}_{i}(t)-\mathbf{r}_{i}(0)\right]^{2}>,
$$

The Green-Kubo relation for the viscosity is given in terms of the pressure auto-correlation function by

$$
\eta=\frac{V}{k T} \int_{0}^{\infty} d t<P_{\alpha \beta}(0) P_{\alpha \beta}(t)>,
$$

where $\alpha \beta=x y, x z, y x, y z, z x$, or $z y$ and for a system of $N$ molecules, the molecular formalism for the pressure tensor is given by

$$
P_{\alpha \beta}=\frac{1}{V}\left[\sum_{i} m_{i} v_{i \alpha} v_{i \beta}+\sum_{i} \sum_{j>i}\left(r_{i \alpha}-r_{j \alpha}\right) F_{i j \beta}\right],
$$

where the indices $i$ and $j$ refer to molecules $i$ and $j$, and $F_{i j}$ is the force between the centers of mass of molecules $i$ and $j$.

Friction constant is obtained from the time integral of the force auto-correlation (FAC) function ${ }^{20,21}$ :

$$
\zeta=\frac{1}{3 k T} \int_{0}^{\tau} \mathrm{dt}\left\langle f_{i}(0) \cdot f_{i}(t)\right\rangle
$$

where $\mathbf{f}_{\mathrm{i}}(\mathrm{t})=\mathbf{F}_{\mathrm{i}}(\mathrm{t})-<\mathbf{F}_{\mathrm{i}}(\mathrm{t})>$ and $\mathbf{F}_{\mathrm{i}}(\mathrm{t})$ is the total force exerted on molecule $i$. Molecular friction force is defined by $\zeta_{\mathrm{M}}=1$ / $\sigma_{\mathrm{r}}=\zeta / \mathrm{M}=\zeta / \mathrm{nm}$ with $\mathrm{M}$ molecular mass of $n$-alkane, $\mathrm{n}$ number of carbons, $\mathrm{m}$ mass of monomer and $\tau_{\mathrm{r}}$ the macroscopic relaxation time of the FAC. ${ }^{21}$

\section{Results and Discussion}

Density. In Figure 1, we plot the density of $n$-alkanes as a function of temperature. The values of the density were obtained from a long time of NpT MD simulations as discussed above. Generally the density decreases linearly with increasing temperature as expected, but an abnormality is found in the long $n$-alkanes at high temperatures. For the short $n$-alkanes, we observed that the volumes of the systems increase infinitely as the temperature increases. This indicates "evaporation" of the systems. However, for the long chain $n$ alkane, the density loses the linear decrement as temperature increases further and becomes flat at high temperatures. This may indicates the existence of a boundary between the small molecular melts and the Rouse regime. In this study, the crossover is between $\mathrm{C}_{44}$ and $\mathrm{C}_{60}$.

Self-diffusion Constant. Self-diffusion constants of $n$ alkanes, D, obtained from the Einstein relation, Eq. (2), are plotted in the inset of Figure 1 as a function of $T$. The dependence of $\mathrm{D}$ on $\mathrm{T}$ in the small molecular melts is obviously different from that in the Rouse regime.

The temperature dependence of the calculated diffusion constants of liquid $n$-alkanes over the whole temperatures considered is suitably described by an Arrhenius plot as shown in Figure 2:

$$
D=D_{0} \exp \left(-E_{D} / R T\right)
$$

where $\mathrm{D}_{0}$ is the pre-exponential factor, RT has the usual meaning, and $\mathrm{E}_{\mathrm{D}}$ is the activation energy of $n$-alkane diffusion. The value of the activation energy is a direct measure of the temperature dependence of self-diffusion constant. The activation energies obtained from the slope of the least square fit are $2.83,3.41,3.84,4.49,4.74,4.87$, and 4.95 $\mathrm{kcal} / \mathrm{mol}$ for $\mathrm{C}_{12}, \mathrm{C}_{20}, \mathrm{C}_{28}, \mathrm{C}_{36}, \mathrm{C}_{44}, \mathrm{C}_{60}$, and $\mathrm{C}_{80}$, respectively. The previous MD simulation study ${ }^{22}$ reported this value as $3.98 \mathrm{kcal} / \mathrm{mol}$ for the UA model of $n$-tetracosane $\left(\mathrm{C}_{24}\right)$ which

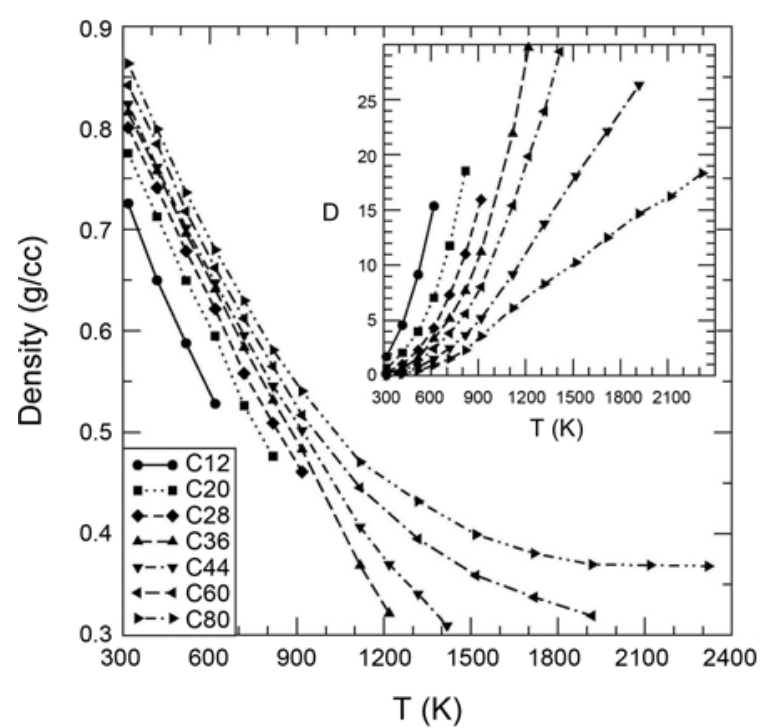

Figure 1. Plot of density $(\mathrm{g} / \mathrm{cc}) v s$. T. The inset shows plot of D $\left(10^{-5} \mathrm{~cm}^{2} / \mathrm{sec}\right) v s$. T. 


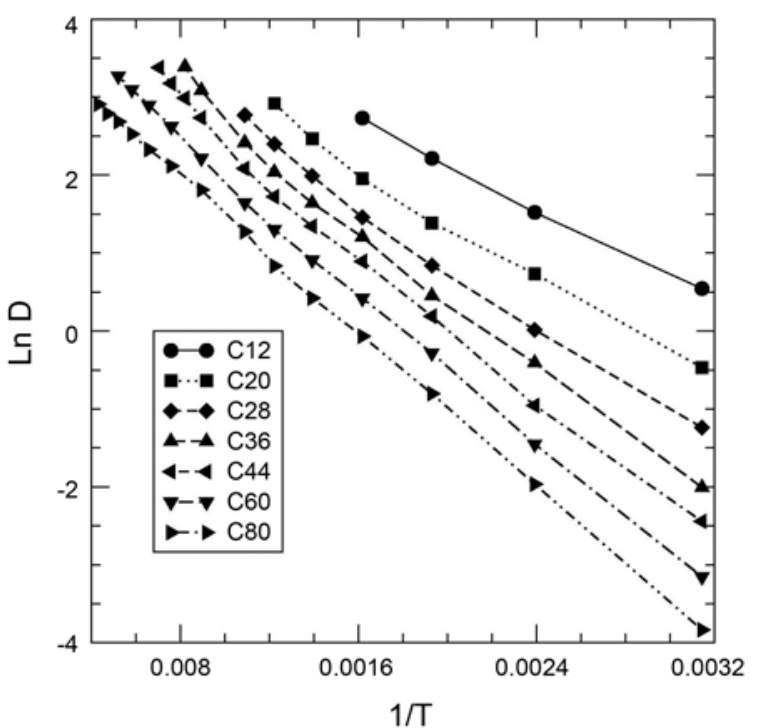

Figure 2. Arrhenius plot of $\mathrm{D}\left(10^{-5} \mathrm{~cm}^{2} / \mathrm{sec}\right) v s .1 / \mathrm{T}$.

is compared with a value of $4.04 \mathrm{kcal} / \mathrm{mol}$ obtained from viscosity measurements ${ }^{23}$ and a value of 4.36 from PFG NMR results. ${ }^{24}$ The values of $\mathrm{E}_{\mathrm{D}}$ are plotted in Figure 3. As chain length $n$ increases the increment of $E_{D}$ decreases, and it is expected to approach an asymptotic value as $n$ increases further. It was reported that $\mathrm{E}_{\mathrm{D}}$ increases linearly with $\log \mathrm{M}$ from $2.32 \mathrm{kcal} / \mathrm{mol}$ for $n$-heptane to $5.81 \mathrm{kcal} / \mathrm{mol}$ for $n$ hexacontane $\left(\mathrm{C}_{60}\right)^{7,25}$ Fleisher ${ }^{5}$ also determined $\mathrm{E}_{\mathrm{D}}$ to be about $4.8 \mathrm{kcal} / \mathrm{mol}$ for several polyethylene independent of molecular weight from 9 to $52.7 \mathrm{~kg} / \mathrm{mol}$, from which we can deduce that $\mathrm{E}_{\mathrm{D}}$ of $n$-alkane reaches an asymptotic limit over $\mathrm{C}_{100}$.

We also show the log-log plot of diffusion constant versus molecular mass in Figure 4. The obtained exponents are between -2.36 and -1.44 . Recent experimental study report-

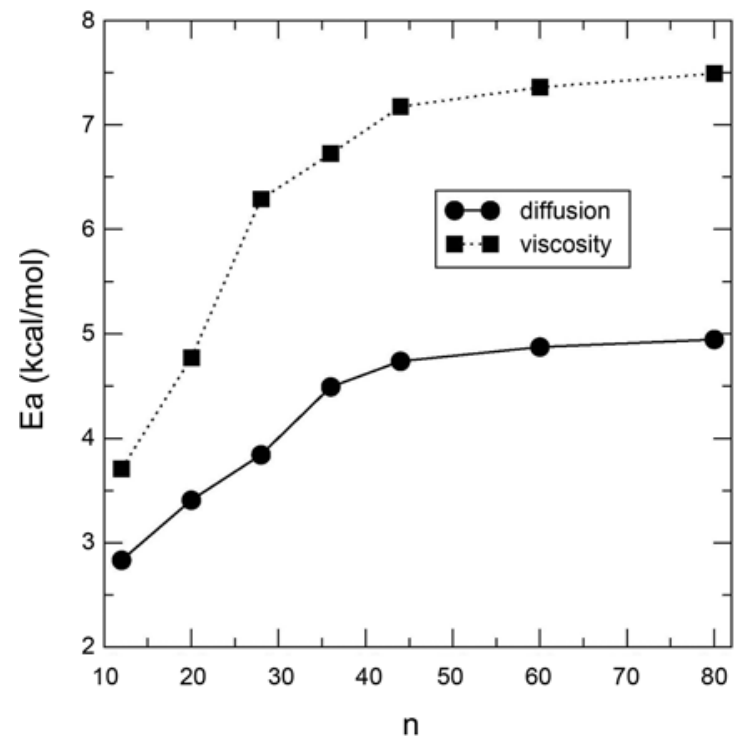

Figure 3. Activation energies of D (circle) and $\eta$ (square) $v s$. chain length $n$.

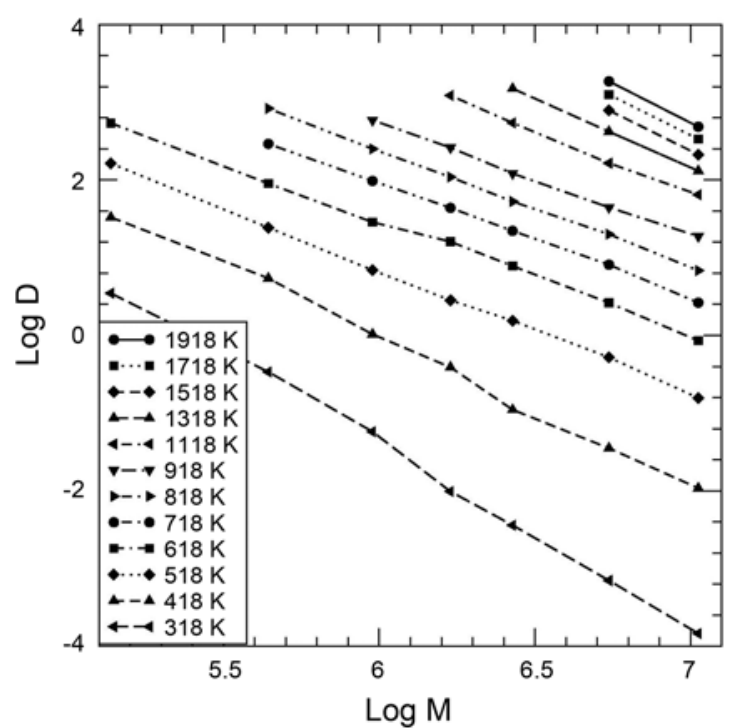

Figure 4. A log-log plot of $\mathrm{D}\left(10^{-5} \mathrm{~cm}^{2} / \mathrm{sec}\right)$ vs. $\mathrm{M}(\mathrm{g} / \mathrm{mol})$.

ed that $\mathrm{D} \sim \mathrm{M}^{-\alpha}$, with a changing approximately linearly from 2.72 to 1.85 as $\mathrm{T}$ increases. ${ }^{7}$ Thus the apparent activation energy also rises linearly with $\log \mathrm{M}$. In the absence of molecular entanglements, Rouse kinetics predicts $\alpha=-1$, but Cohen-Tumbull-Bueche free-volume effects due to molecular chain ends add a further nonpower-law term, ${ }^{26}$ enhancing D increasingly at low $\mathrm{M}$.

Viscosity. Viscosities of $n$-alkanes, $\eta$, are obtained from the Green-Kubo relation. It is worth note that the pressure auto-correlation functions of long chains of $n$-alkanes at low temperatures have the non-decaying long-time tails (not shown) and that it is impossible to get the plateau values in the running time integrals of the pressure auto-correlation functions. For example, in this study, $\mathrm{C}_{60}$ at $318 \mathrm{~K}$ and $\mathrm{C}_{80}$ at $318 \mathrm{~K}$ and $418 \mathrm{~K}$. The temperature dependence of the calculated viscosities of liquid $n$-alkanes over the temper-

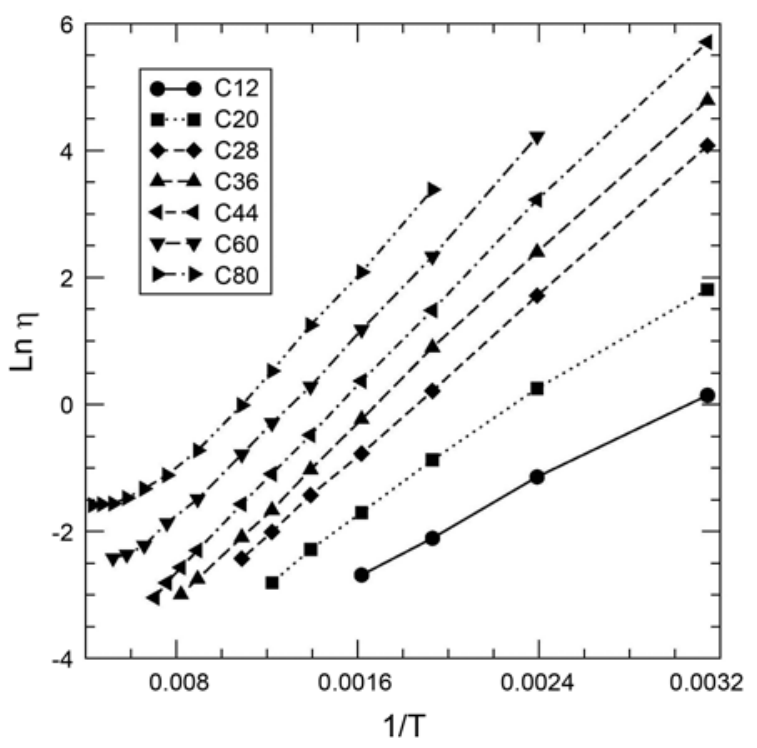

Figure 5. Arrhenius plot of $\eta(\mathrm{cP}) v s .1 / \mathrm{T}$. 


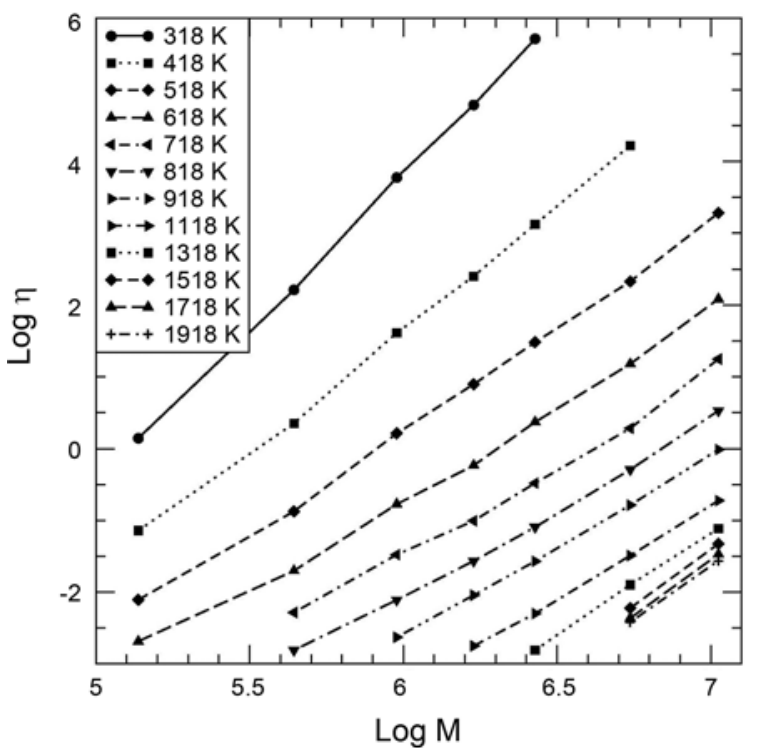

Figure 6. A log-log plot of $\eta(\mathrm{cP}) v s . \mathrm{M}(\mathrm{g} / \mathrm{mol})$.

atures is also suitably described by an Arrhenius plot except very high temperatures as shown in Figure 5 :

$$
\eta=\mathrm{C}_{0} \exp \left(\mathrm{E}_{\eta} / \mathrm{RT}\right)
$$

where $\mathrm{C}_{0}$ is the pre-exponential factor and $\mathrm{E}_{\eta}$ is the activation energy of $n$-alkane viscosity. But the experimental result for the linear polyethylene ${ }^{6}$ showed that the lines are slightly curved, indicating that the temperature dependence, as in most polymers, does not follow the Arrhenius equation. The activation energies obtained from the slope of the least square fit except $\mathrm{T}$ over $1318 \mathrm{~K}$ are $3.71,4.77,6.29,6.72$, $7.17,7.36$, and $7.49 \mathrm{kcal} / \mathrm{mol}$ for $\mathrm{C}_{12}, \mathrm{C}_{20}, \mathrm{C}_{28}, \mathrm{C}_{36}, \mathrm{C}_{44}, \mathrm{C}_{60}$, and $\mathrm{C}_{80}$, respectively and are plotted in Figure 3. It was experimentally reported for $n$-alkanes and linear polyethyl$e^{6} e^{6}$ that the activation energy increases with chain length and at the highest molecular weight tested $(\mathrm{M} \approx 4.4 \mathrm{~kg} / \mathrm{mol})$ the activation energy reaches $6.6 \mathrm{kcal} / \mathrm{mol}$, which is similar to the average value found for NBS $1482-4(6.7 \mathrm{kcal} / \mathrm{mol})$ and the values reported by others ${ }^{27,28}$ for high molecular weight linear polyethylene $(6.1-6.9 \mathrm{kcal} / \mathrm{mol})$.

Figure 6 shows the log-log plot of viscosity versus molecular mass. The obtained exponents are between 2.41-3.32 except $\mathrm{C}_{12}$ (4.32). The experimental results for $n$-alkanes and linear polyethylene $e^{6}$ show that $\eta$ is well described by the power law $\eta \sim \mathrm{M}^{1.8}$ at low molecular weight $(\mathrm{M}<5 \mathrm{~kg} / \mathrm{mol})$ and $\eta \sim \mathrm{M}^{3.64}$ at high molecular weight $(\mathrm{M}>5 \mathrm{~kg} / \mathrm{mol})$ at $448 \mathrm{~K}$. The molecular weight of the $n$-alkanes tested in this study is much lower than this experimental range of molecular weight, but the lowest exponent gives 2.41 at $818 \mathrm{~K}$.

Friction Constant. It is well known that the calculation of friction constant from the force auto-correlation function is fairly hard due to the non-decaying long-time tails. As Kubo pointed out in his "fluctuation-dissipation theorem", ${ }^{19}$ the correlation function of random force $\mathrm{R}$ will decay in a time interval of $\tau_{\mathrm{c}}$ (microscopic time or collision duration time), whereas that of the total force $\mathrm{F}$ has two parts, the short time

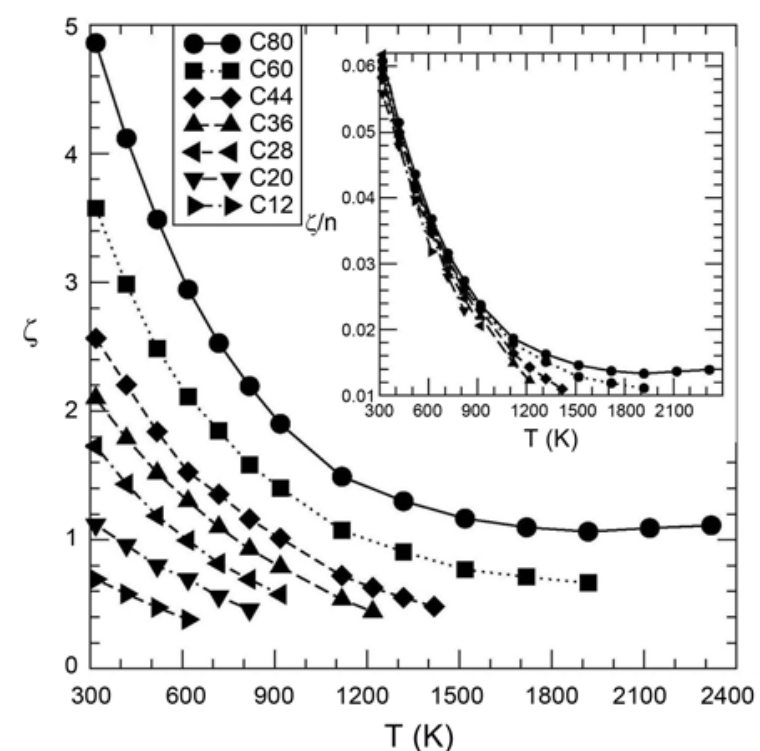

Figure 7. Friction constants, $\zeta\left(10^{3} \mathrm{~g} / \mathrm{ps} \cdot \mathrm{mol}\right) v s$. T. The inset shows plot of $\zeta / n v s$. T.

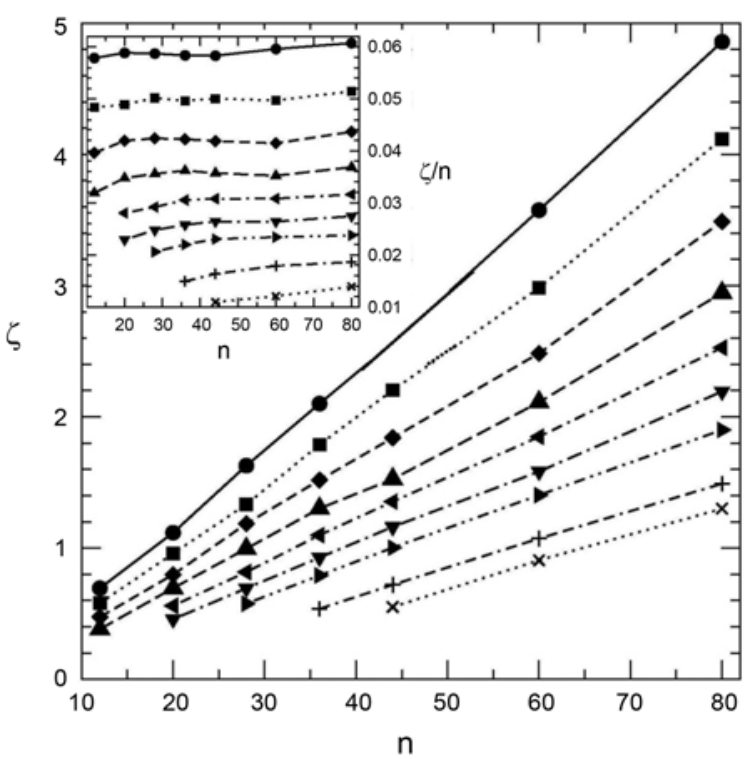

Figure 8. Friction constants, $\zeta\left(10^{3} \mathrm{~g} / \mathrm{ps} \cdot \mathrm{mol}\right) v s$. chain length $n$. The inset shows plot of $\zeta / n v$ s. $n$. From top, $\mathrm{T}=318,418,518,618$, $718,818,918,1118$ and $1318 \mathrm{~K}$, respectively.

or the fast part similar to that of the random force and the slow part which should just cancel the fast part in the time integration. ${ }^{29}$ This means that the time integral of Eq. (4) up to $\tau=\infty$ is equal to zero.

The time integral in Eq. (4) attains a plateau value for $\tau$ satisfying $\tau_{\mathrm{c}}<<\tau<<\tau_{\mathrm{r}}$, if the upper limit of the time integral, Eq. (4), is chosen that $\tau_{\mathrm{c}}<<\tau<<\tau_{\mathrm{r}}$ because the slow tail of the correlation function is cut off. However, we were unable to get the plateau value in the running time integral of the force auto-correlation function. Kubo suggested that the friction constants should be obtained from the random FAC function not from the total FAC and that there exists a difficulty to separate the random force part from the 
total force. We could obtain the friction constants by the time integral of the total FAC with choosing the upper limit of $\tau$ as the time that the FAC has the first negative value by assuming that the fast random force correlation ends at that time.

Figure 7 shows the plot of $\zeta v s$. T for given $n$-alkanes. The calculated friction constants give a correct qualitative trend: $\zeta$ decreases with increasing T. However, the abnormality in density of the long $n$-alkanes at high temperatures is recovered in the friction constant. For the short $n$-alkanes, $\zeta$ is inversely proportional to $\mathrm{T}$ but for the long chain $n$-alkane, the density loses the inverse decrement as temperature increases further and becomes flat at high temperatures. When $\zeta / n$ is plotted as a function of $\mathrm{T}$ as shown in the inset of Figure 7, the overall behavior of $\zeta / n$ for all $n$-alkanes is rather coincided except at high temperatures.

We plot $\zeta$ vs. $n$ in Figure 8 in which $\zeta$ increases linearly with increasing $n$ for a given T. The slope of $\zeta$ vs. $n$ decreases with increasing T. An atomistic MD simulation of linear polyethylene melts ${ }^{30}$ reported that the friction constant extracted from D by applying the Rouse model is seen to increase from value of short $n$-alkanes to a chain-lengthindependent plateau one, reached in a region of $n=60-80$. The linear increase of $\zeta$ with increasing $n$ as shown in Figure 8 might indicate that the liquid $n$-alkanes over the whole temperatures considered are far away from the Rouse regime. When $\zeta / n$ is plotted as a function of $n$ as shown in the inset of Figure $8, \zeta / n$ is almost independent of $n$ for given temperatures, which indicates the friction is governed by the local segmental friction not by the topological entanglement effect.

\section{Conclusion}

We have presented the results for viscosity and selfdiffusion constants of model systems for liquid $n$-alkane oligomers $\left(\mathrm{C}_{12} \sim \mathrm{C}_{80}\right)$ at high temperatures $(2300 \mathrm{~K})$ using molecular dynamics (MD) simulations in a canonical ensemble. The long chains of these $n$-alkanes at high temperatures show an abnormality in density. Calculated viscosity $\eta$ and self-diffusion constants D are comparable with experimental results and the temperature dependence of both $\eta$ and D is suitably described by the Arrhenius plot. The behavior of both activation energies, $E_{n}$ and $E_{D}$, indicates that the activation energies approach asymptotic values with increasing chain length, which is consistent with the experimental observation. The calculated monomeric friction constant $\zeta$ shows an abnormality again as in density. The slope of $\zeta v s . n$ decreases with increasing $\mathrm{T}$ but does not provide a chain-length-independent plateau.

Acknowledgments. This research was supported by a Kyungsung University Research Grant in 2011.

\section{References}

1. Berry, G. C.; Fox, T. G Adv. Polym. Sci. 1968, 5, 261.

2. Fox, T. G.; Flory, P. J. J. Phys. Colloid Chem. 1951, 55, 221.

3. Lodge, T. P.; Rotstein, N. A.; Prager, S. Adv. Chem. Phys. 1990, 9 , 1.

4. Ferry, J. D. Viscoelastic Properties of Polymers; 3rd ed.; Wiley: New York, 1980.

5. Fleisher, G. Polym. Bull. (Berlin) 1983, 9, 152

6. Pearson, D. S.; Ver Strate, G.; von Meerwall, E.; Schilling, F. C. Macromolecules 1987, 20, 1133 .

7. Von Meerwall, E.; Beckman, S.; Jang, J.; Mattice, W. L. J. Chem. Phys. 1998, 108, 4299.

8. Tirrell, M. Rubber Chem. Technol. 1984, 57, 523.

9. De Gennes, P.-G. Scaling Concepts in Polymer Physics; Cornell University Press: Ithaca, New York, 1979.

10. Mundy, C. J.; Siepmann, J. I.; Klein, M. L. J. Chem. Phys. 1995, 102,3376 .

11. Cui, S. T.; Cummings, P. T.; Cochran, H. D. J. Chem. Phys. 1996, 104, 255.

12. Cui, S. T.; Gupta, S. A.; Cummings, P. T.; Cochran, H. D. J. Chem. Phys. 1996, 105, 1214.

13. Evans, D. J. J. Chem. Phys. 1983, 78, 3297.

14. Brown, D.; Clarke, J. H. R. Mol. Phys. 1984, 51, 1243.

15. Jorgensen, W. L.; Madura, J. D.; Swenson, C. J. J. Am. Chem. Soc. 1984, 106, 6638.

16. Gear, C. W. Numerical Initial Value Problems in Ordinary Differential Equation; Prentice-Hall, Englewood Cliffs, NJ, 1971.

17. Andersen, H. J. Comput. Phys. 1984, 52, 24.

18. Allen, M. P.; Tildesley, D. J. Computer Simulation of Liquids; Oxford: Oxford Univ. Press, 1987; p 81.

19. McQuarrie, D. A. Statistical Mechanics; Harper and Row: New York, 1976.

20. Ciccotti, G.; Ferrario, M.; Hynes, J. T.; Kapral, R. J. Chem. Phys. 1990, 93, 7137.

21. Kubo, R. Rep. Prog. Phys. 1966, 29, 255.

22. Mondello, M.; Grest, G. S. J. Chem. Phys. 1995, 103, 7156.

23. Nederbragt, G. W.; Boelhouwer, J. W. M. Physica 1947, 13, 305.

24. See page 7161 in Reference 22.

25. Ertl, H.; Dullien, F. A. L. AIChE J. 1973, 19, 1215.

26. Cohen, M. H.; Tumbull, D. J. Chem. Phys. 1959, 31, 1164.

27. Mendelson, R. A.; Bowles, W. A.; Finer, F. L. J. Polym. Sci., Part A-2, 1970, 8, 105

28. Raju, V. R.; Smith, G. G.; Marin, G.; Knox, J. R.; Graessley, W. W. J. Polym. Sci., Polym. Phys. Ed. 1979, 17, 1183.

29. Kubo approximately described these two force auto-correlation functions in his original papers, see Fig. 2 in Ref. 21.

30. Harandaris, V. A.; Mavrantzas, V. G.; Theodorou, D. N. Macromolecules 1998, 31, 7934. 